Revue de l'Institut des langues et cultures

d'Europe, Amérique, Afrique, Asie et Australie

$21 \mid 2015$

Discours politique et culturel dans la Russie

contemporaine

\title{
Le second degré entendu comme réflexivité dans quelques contes d'Europe centrale et orientale
}

Second Degree as Reflexivity in Selected Central and Eastern Europe Folktales

Anne-Marie Monluçon

\section{OpenEdition}

\section{Journals}

Édition électronique

URL : http://journals.openedition.org/ilcea/3082

DOI : 10.4000/ilcea.3082

ISSN : 2101-0609

Éditeur

UGA Éditions/Université Grenoble Alpes

Édition imprimée

ISBN : 978-2-84310-294-3

ISSN : 1639-6073

Référence électronique

Anne-Marie Monluçon, «Le second degré entendu comme réflexivité dans quelques contes d'Europe centrale et orientale », ILCEA [En ligne], 21 | 2015, mis en ligne le 01 février 2015, consulté le 01 mai 2019. URL : http://journals.openedition.org/ilcea/3082 ; DOI : 10.4000/ilcea.3082

Ce document a été généré automatiquement le 1 mai 2019.

(C) ILCEA 


\title{
Le second degré entendu comme réflexivité dans quelques contes d'Europe centrale et orientale
}

\author{
Second Degree as Reflexivity in Selected Central and Eastern Europe Folktales
}

\author{
Anne-Marie Monluçon
}

1 L'un des succès de librairie depuis 1998 et l'un des albums les plus lus dans les petites classes, en France, n'est autre que Le Loup sentimental de Geoffroy de Pennart (1998). Ce livre raconte l'histoire d'un jeune loup qui reçoit de son père, au moment où il quitte la maison, la liste des proies qu'il est autorisé à manger : la chèvre et ses chevreaux, le Petit Chaperon rouge, les trois petits cochons, Pierre (le héros du conte musical de Prokofiev, Pierre et le Loup), le Petit Poucet et ses frères... Hélas, à chaque fois que le héros croise l'une de ses victimes potentielles, celui-ci se laisse attendrir. Il n'évite de mourir de faim qu'en dévorant l'ogre qui détient le Petit Poucet et ses frères. Encore faut-il préciser que l'ogre l'a rendu fou de rage en le traitant avec mépris. Or ce conte moderne présente une structure commune avec les contes-types AT 122A et 122M (Aarne \& Thompson, 1961) dont le canevas se résume comme suit : affamé, le loup rencontre différents animaux qu'il veut manger. Il se fait berner par chacun d'eux. Pour finir, il se contente d'une charogne. Ce schéma se retrouve dans les contes 36 et 37 des Contes populaires russes d'Afanassiev $(2010)^{1}$, intitulés respectivement « Le Loup idiot » I et II. Dans la première variante, le loup laisse échapper un chien, un bouc, une truie et ses pourceaux; dans la seconde, une jument, un bélier et un tailleur dont la ruse et le courage ne sont pas sans rappeler ceux du vaillant petit tailleur des frères Grimm². Il n'est pas certain que les contes d'Afanassiev soient la source qui a inspiré Geoffroy de Pennart, car les contestypes auxquels son album se rattache sont connus du monde entier. Mais il est remarquable que le passage du conte populaire à l'album pour enfants se caractérise par deux transformations principales : la suppression des sous-entendus sexuels (dans « Le Loup idiot » II, le tailleur arrache la queue du loup) et l'introduction du second degré. En effet, le jeune lecteur du Loup sentimental doit, tout comme le loup, être assez cultivé pour reconnaître les proies, issues de différentes traditions nationales du conte et du 
conte musical ${ }^{3}$. Le second degré se présente ici sous la forme d'une réécriture ou d'un conte détourné, mais aussi comme réflexivité, car cette parodie des contes traditionnels donne à réfléchir sur les lois du genre et les possibilités de s'en affranchir, en mangeant ou en écrivant autre chose que prévu... L'une des questions que posent ces exemples est la suivante: le second degré est-il une invention de la littérature pour la jeunesse contemporaine ou est-il déjà présent dans ses sources populaires pour adultes ou pour « tout public »? L'autre question porte sur la manière dont les conteurs ont introduit le second degré dans les contes et ses répercussions sur leur signification.

2 Le second degré peut s'entendre au sens de réflexivité, c'est-à-dire comme l'un des aspects du fonctionnement auto-référentiel de la littérature, lorsque celle-ci ne se contente plus de faire référence au monde mais fait référence à elle-même. De nombreux contes réfléchissent soit directement sur eux-mêmes, soit sur le genre du conte, soit plus largement sur la littérature en général. La présence d'une forte réflexivité dans le conte, genre réputé populaire et récemment dédié à la jeunesse, est paradoxale car elle est généralement associée à la littérature savante. On retrouve ainsi une réflexion sur l'art de lire ou de conter, dans six recueils de contes d'Europe centrale ou orientale. Les Contes de Pologne 4 ont été publiés en 1990 chez Gründ. Les Contes polonais $(2007)^{5}$ et les Contes yiddish $(2000)^{6}$, publiés à l'École des loisirs, sont explicitement destinés au jeune public. Des contes réflexifs se rencontrent également dans les Contes pour les enfants et la maison des frères Grimm ${ }^{7}$, dans les Contes populaires russes d'Afanassiev ainsi que dans le recueil « tout public » intitulé Le Rameau de l'arbre du soleil ${ }^{8}$. Il s'agit, dans ce dernier, de contes tsiganes polonais publiés chez Wallâda, éditeur militant.

Il s'agira de comprendre ce que ces contes réflexifs ont à dire sur la littérature, mais aussi comment ils le disent. On peut distinguer un premier mode d'insertion de la réflexivité, à la périphérie des textes, avec des figures de conteurs en position liminaire dans les recueils ou dans les contes pris à l'unité, ainsi que des figures de lecteurs au second plan du récit (I). Mais les contes où le second degré se greffe sur le premier degré sont plus nombreux. L'art de raconter ou de se taire, car les deux vont de pair, ainsi que l'art de lire, au sens d'interpréter, remplissent alors une fonction dramatique absolument centrale et sont intimement liés à l'action principale. Ce cas de figure correspond au second mode d'insertion de la réflexivité (II). Cependant on ne peut se contenter de décrire en quoi tel héros conteur ou lecteur fait progresser l'action. En définitive, sur quelle sagesse débouche la réflexivité dans nos contes? Le fonctionnement autoréférentiel de nos contes ne nous ramène-t-il pas à des questions concernant la vie ? Plus précisément, les personnages experts en matière de récit ou d'interprétation ne sont-ils pas souvent des experts dans le déchiffrement du désir de l'Autre ou des secrets de la sexualité (III)?

\section{La réflexivité à la périphérie du conte}

\section{I.1. Figures de conteurs en ouverture et clôture des recueils}

4 Une première série de contes met en scène d'une part des figures de conteurs en position liminaire et d'autre part des doubles du lecteur/auditeur à la périphérie du récit. Deux des cinq recueils commencent par un conte racontant comment le protagoniste est devenu conteur. Détail révélateur, le récit inaugural des Contes de Pologne n'est pas présenté comme un conte mais comme une introduction au recueil. Il raconte comment 
un soldat démobilisé, pauvre mais charitable, reçoit en récompense de sa bonté le don surnaturel de jouer du violon et de raconter des histoires. Le mendiant qui lui octroie ce don ne fait que conforter sa vocation qui s'exprime dans les termes suivants :

Aussi aimerais-je apprendre à égayer les autres [...]. Lorsque j'étais à l'armée, nous traversions maintes villes et maints villages et nous avons vécu toutes sortes d'aventures. Ainsi j'ai récolté beaucoup d'histoires et d'anecdotes. [...] Mes biens à moi ne sont que des aventures secrètes pleines d'inexplicable magie mais également des histoires humaines, des joies et des tourments. (C.D.P. : 10)

En même temps que sa vocation, le personnage propose une véritable définition du genre qu'il va servir, où l'on reconnaît la diversité d'un répertoire empruntant au conte facétieux, qui « égaye », à l'anecdote, tirée de l'expérience vécue, au merveilleux avec le recours au surnaturel. L'excipit de cette «introduction» est si explicite et pédagogique que l'on soupçonne l'adaptateur de l'avoir inventée, plutôt qu'empruntée à la tradition orale :

Et il s'en fut de village en village et de ville en ville afin de réjouir les gens non seulement avec son violon, mais aussi avec les histoires et la bonne humeur qu'il trouvait en lui-même. Aujourd'hui encore, il réjouit ses auditeurs grâce à son pouvoir magique. Il les émeut et les contraint à réfléchir à la destinée de l'homme ${ }^{9}$. ( C.D.P. : 12)

6 Le même soupçon pèse sur le premier des contes tsiganes polonais intitulé « Le Conte des contes ». Le narrateur qui dit « je » - c'est assez rare pour le signaler - part en quête de contes et en trouve grâce à des nomades, et notamment grâce à une belle Tsigane surnommée "Sœur des oiseaux». Tout suggère qu'il s'agit d'une transposition de l'autobiographie du collecteur, Jerzy Ficowski. Poète et traducteur gadjo, c'est-à-dire nonTsigane, celui-ci a voyagé avec des Tsiganes et collecté leurs contes entre 1948 et 1950 ( Études tsiganes, 2013 : 59). Dans le même recueil, le conte « Les douze chevaux et la jeune fille de l'île flottante » occupe la vingt-neuvième place sur trente-six, c'est-à-dire qu'il occupe l'autre position liminaire, celle de clôture et non plus d'ouverture du livre. On y retrouve ce narrateur qui dit « je » et n'est pas présenté comme un Tsigane, comme celui $\mathrm{du}$ conte inaugural et contrairement à tous les autres personnages du recueil. Celui-ci évoque la disparition de l'univers des contes avec la mort du "conteur de contes", «le plus vieux des Tsiganes ", et l'arrêt de son convoi qui se sédentarise dans un village de montagne (R.A.S. : 189). Les contes dont le héros est un conteur sont tous ici en relation soit avec le rassemblement du recueil soit avec le passage à l'écrit. Ils semblent avoir un rapport avec la mort du conte, au sens de la disparition de sa transmission orale.

\section{I.2. Figures du conteur dans l'excipit des contes}

Outre la position liminaire d'ouverture ou de clôture des recueils, le personnage du conteur apparaît aussi dans l'excipit de nombreux contes ${ }^{10}$. Ces excipits sont mémorables parce qu'ils sont à la fois comiques et difficiles à interpréter. La tentation est donc de les trouver simplement absurdes, dépourvus de toute signification. Dans La Tradition orale russe, Lise Gruel-Apert (1995:126) traite ce point très rapidement, en leur prêtant une fonction de détachement du conteur par rapport à son récit et de retour au réel. Comme certaines de ces formules de conclusion se retrouvent, presque identiques, à la fin de contes différents, il est tentant de les considérer comme à la fois interchangeables et déconnectées de l'intrigue et du sens du conte qu'elles concluent. Toutefois dans le conte « Si ça ne te plaît pas, n'écoute pas » (C.P.R., t. 3, nº $285:$ 296-298), l'excipit formulaire, 
évoquant un conteur privé de boisson et miel au banquet donné par le héros, se retrouve fortement lié à l'action principale par une relation d'inversion. Le héros Ivan s'offre, avec l'argent du vol, ce dont est privé le conteur: «Le voilà avançant cahin-caha, tout en trempant son pain d'épice dans le miel et en l'enfournant dans sa bouche.» (C.P.R., t. 3 : 296) Or ce (faux) dénouement intervient au début du conte, avant même que le pickpocket Ivan ne se transforme en conteur si habile qu'il parvient à gagner un pari très risqué conclu avec le « seigneur du lieu ». À l'inverse, le conteur, pourtant virtuose, pour avoir réussi un somptueux récit réflexif avec enchâssement, se présente sous les traits traditionnels du minable frustré, en des termes qui anticipent presque mot pour mot l'excipit du conte suivant « Foma Berénikov » (C.P.R., t. 3, nº 286 : 298-301) :

J'y étais, du miel et de la vodka j'ai voulu boire, sur ma moustache ils ont coulé, dans ma bouche rien n'est tombé. On m'a donné un bonnet et on m'a poussé par le collet ; on m'en a donné un autre et je l'ai jeté sous le portail ! Voici la fin du conte, donnez-moi un pot de miel ! (C.P.R., t. $3: 298$ )

Plusieurs excipits ont en commun d'affirmer, comme celui-ci, la continuité ou la proximité entre le monde fictif et le monde réel, à travers la figure du conteur qui circule entre les deux. Mais cette tentative d'accréditer le récit se disqualifie à la fois par le comique et l'invraisemblance. Voici l'épilogue du conte « Les dons du bon génie » dans Les Contes de Pologne:

On chassa le traitre serviteur du château et le soldat put célébrer ses noces avec la princesse. J'y étais... J'y ai mangé maints gâteaux et bu force boissons. On tira des coups de canon en l'honneur du nouveau souverain. On me prit pour un boulet et c'est pourquoi je suis retombé ici, parmi vous. (C.D.P. : 84)

L'outrance comique d'un narrateur qui fait bombance avant d'être expulsé de l'univers du conte aboutit sans doute au même résultat que les excipits qui évoquent, à l'inverse, la frustration du narrateur : dans le premier cas, l'invraisemblance fait que l'on ne croit pas qu'il soit possible de prendre part à la féérie du conte, dans le second, le contraste, l'irrémédiable séparation des deux univers est explicite :

J'y étais, du miel et de la bière j'ai voulu goûter, sur ma moustache ils ont coulé, dans ma bouche rien n'est tombé. J'ai voulu manger du chou, mais mon ventre est resté creux. On m'a donné un bonnet et dehors on m'a chassé. On m'a donné un fouet de pois et une bride de navets. J'ai vu un paysan qui faisait du feu pour sécher son grain, j'ai attaché près de là ma rosse, elle a fondu, les poules ont becqueté le fouet et les gorets ont mangé la bride ! (C.P.R., t. $3: 301)^{11}$

On peut tenter plusieurs interprétations de cet excipit de "Foma Berenikov" (conte 286). Tout d'abord, de manière superficielle, la frustration du conteur peut-être un discret appel du pied pour que l'auditoire lui paye un coup à boire. À un autre niveau, on note que la désintégration des accessoires du conteur prolonge la logique du conte, comme si le surnaturel débordait dans le réel, mais pour mieux s'autodétruire et proclamer sa fin. Enfin, ce dénouement où le conteur (et peut-être l'auditeur) passent si près de la complète félicité ressemble au "travail du rêve " analysé par Freud (Belmont, 1999, chap. 3). Alors que le conte, comme le rêve, exprime toujours la satisfaction d'un désir - ici féerie sociale, érotique -, il reste malgré tout une «formation de compromis » dans laquelle la censure joue un rôle, en nous réveillant parfois avant la pleine satisfaction de notre désir. Mais outre ce que de tels dénouements disent du rapport des contes avec nos désirs et de leur fonction compensatoire, ils proposent en même temps une ébauche de réflexion sur le genre. Tout d'abord, il n'est pas anodin que le conteur apparaisse généralement, dans ces excipits, comme un anti-héros, une Cendrillon au masculin dont le carrosse redevient citrouille à minuit. Ce peut être une manière de 
s'assumer comme serviteur d'un genre mineur et populaire. En second lieu, la continuité entre fiction et réel invoquée par le conteur prend sens si l'on comprend qu'elle n'est pas à prendre au sens littéral. Le lien entre le passé éloigné de l'action du conte et le présent de la narration peut signifier que c'est la portée symbolique du conte qui est toujours actuelle, vivante, et non pas son univers ou ses personnages. Enfin, le fait que les excipits résistent à l'interprétation constitue la plus vigoureuse des injonctions à en chercher le sens et le paradigme de la lecture d'un genre dont la force réside, comme l'écrit Walter Benjamin (2011/1936), dans le refus de fournir des explications, ce qui ne veut pas dire que l'on ne peut pas en trouver, mais que le texte laisse au lecteur du champ pour mener à bien lui-même un travail d'interprétation.

\section{I.3. Figures du lecteur à la périphérie du récit}

Toutefois, même si le conte permet une grande liberté d'interprétation, chaque fois qu'il comporte en son sein une figure de lecteur, il livre en même temps tout ou partie de son mode d'emploi, il guide le lecteur réel grâce au lecteur fictif, qui est parfois un repoussoir. Ainsi dans le conte yiddish "La chèvre de Chelm » avons-nous deux couples de lecteurs anti-thétiques. Il s'agit d'une farce mécréante qui se moque des dévots du hassidisme. Au commencement, le rabbin de Chelm tombe malade et le médecin prescrit de le soigner avec du lait de chèvre. Ses deux "plus fervents hassidim " c'est-à-dire disciples, sont chargés d'acheter une chèvre pour lui à la campagne. Mais ces deux dévots aiment la boisson et s'arrêtent dans un établissement dont l'aubergiste échange subrepticement la chèvre aux pis gorgés de lait contre un bouc. La femme du rabbin, après expertise d'un paysan, renvoie les étudiants chez le propriétaire de la chèvre. Au retour, l'aubergiste restitue la chèvre, si bien que les deux hassidim se ridiculisent lorsqu'ils viennent protester auprès du propriétaire. Ce scénario se répète autant de fois que les deux dévots font l'aller et le retour, puisqu'à chaque fois, ils sont pris d'une soif intense à l'approche de l'auberge. Le subterfuge de l'aubergiste, met définitivement en échec la sagacité des hassidim et du rabbin, qui conclut à un prodige surnaturel : chaque fois que la «chèvre met le sabot dans [leur] ville, elle se transforme en bouc » (C.Y. : 66). En première lecture, ce conte drolatique et rationaliste vise deux cibles traditionnelles : la ville de Chelm qui, dans la tradition juive, est le "vivier des imbéciles ", "la ville des nigauds ", puis les dévots du hassidisme trop prompts à croire aux miracles. Mais à un second niveau, nous avons affaire à un conte philosophique et réflexif consacré au bon usage du temps dans la vie et dans la conduite du récit. La réflexion sur le temps est lancée avec une grande maladresse par les deux étudiants en théologie. En bons pédants et machistes, ils font la leçon à l'épouse du rabbin : « Ne sois pas si impatiente, Malka, prends le temps qu'il faut. Il est dit dans la Torah : "Il faut faire ce que l'on doit faire avec discernement." » (C.Y. : 53) ${ }^{12}$ La bêtise de ces deux lecteurs prétentieux éclate. Tout d'abord, leur leçon est incohérente, car ils introduisent le thème du rapport au temps, puis font une citation concernant le discernement, ce qui est pour ainsi dire un hors-sujet par rapport à leur propos, à moins d'établir un lien logique entre patience et capacité de jugement. Ensuite, rapporté à la situation, leur conseil est un contre-sens, car même si Malka fait preuve de persévérance, le bouc ne donnera pas de lait. Cette réplique est surtout comique parce que dans tout ce qui précède ou qui suit, les deux compères ne savent pas user du temps avec discernement. Ils sont tantôt trop pressés de célébrer leur succès en s'arrêtant boire à l'auberge avant d'avoir livré la chèvre au rabbin, tantôt à cause de leur ivrognerie et de leur sottise, trop lents à secourir celui-ci. Ces deux hassidim sont typiquement deux 
piètres lecteurs, qui n'ont rien su tirer de valable de la Torah. Dans ce contexte, le narrateur nous livre les réactions de la chèvre aux allers et retours qu'on lui impose :

Elle [la chèvre] commençait à se fatiguer mais elle avait le pressentiment que son chemin allait s'arrêter un peu plus loin, à l'auberge, elle n'aurait pas beaucoup à avancer.

Il fut ainsi qu'elle l'avait compris: les chèvres élevées au contact des hommes peuvent anticiper leurs actions. (C.Y.: 64)

Pourquoi ces lignes nous font-elles sourire? Parce que nous, lecteurs, nous reconnaissons dans les réactions de la chèvre. L'anthropomorphisme dont use le narrateur ainsi que sa maxime malicieuse nous autorisent à voir en celle-ci une figure du lecteur qui pointe le risque d'ennui ainsi que la trop grande prévisibilité du scénario lorsque l'on abuse du procédé de la répétition. Ce faisant, le narrateur déplace le thème du bon usage du temps de la vie à l'art du récit, en pointant les forces et les faiblesses de son propre conte. Le comique de répétition est une force qui repose sur la lenteur. Mais pour bien en user, pour éviter l'ennui du lecteur, il faut aussi pratiquer l'art de la variation et de l'accélération, ne pas répéter le scénario strictement à l'identique. En d'autres termes, il faut savoir changer de rythme à bon escient, ce qu'accomplit fort bien ce conteur tout au long de " La Chèvre de Chelm ». Rapporté à l'usage du temps dans la vie et à la réflexion ratée des deux hassidim, ce conte critique la leçon de sagesse trop facile qui consiste à ne conseiller que la patience. Il suggère en revanche qu'il faut éviter une application automatique des conseils de sagesse et s'adapter aux circonstances: tantôt ralentir, tantôt accélérer, comme dans tout beau récit. Autrement dit, dans cet exemple, les figures de bons et mauvais lecteurs incluses dans la fiction constituent un moyen de guidage assez précis du lecteur réel et limite la liberté d'interprétation évoquée plus haut.

\section{La greffe du second degré sur l'action principale}

\section{II.1. L'art de conter, clé de la réussite du héros}

Dans «L'idiot qui épousa la princesse ", tiré du recueil Contes polonais, tout commence de manière traditionnelle. Un roi promet sa fille à celui qui parviendra à escalader une montagne de diamant. L'idiot se lance dans cette entreprise après l'échec des prétendants de la noblesse et de ses deux aînés, présentés comme "intelligents ». Il se procure un objet magique, un pipeau, au terme d'une seule épreuve qualifiante qui consiste à faire preuve de compassion envers un mendiant. Grâce à ce pipeau, il triomphe des trois épreuves principales qui suivent. Concrètement, cela veut dire que notre protagoniste réussit à escalader la montagne de diamant, à réunir tous les agneaux dorés de la terre en douze heures, puis tous les lièvres du monde en dix-huit heures. C'est alors que la mauvaise foi de la noblesse éclate : la princesse est furieuse de faire une mésalliance et les prétendants nobles convainquent le roi de tendre un piège à l'idiot. Il s'agit de demander au héros de fournir un lièvre pour le repas de noces, pour l'accuser aussitôt de donner ce qui ne lui appartient pas, autrement dit, pour l'accuser d'être un voleur. Ce complot amène cinq épreuves identificatoires ${ }^{13}$. Averti on ne sait comment, l'idiot renvoie le premier ambassadeur qu'on lui adresse, à savoir le cuisinier, puis donne un lièvre, en échange d' " un présent de valeur ", aux trois femmes qui se présentent successivement à lui, la cuisinière, la reine et enfin la princesse. Mais à chaque fois, il joue ensuite du pipeau et le lièvre s'échappe. En désespoir de cause, le roi se présente en personne, et cette fois le narrateur nous dévoile ce qu'exige l'idiot en échange du lièvre : le roi doit 
embrasser la vieille rosse de l'idiot sur le cul. Celui-ci s'exécute. Alors que le héros vient de triompher de ces cinq épreuves, ses rivaux lui tendent un ultime piège ${ }^{14}:$ "Dîtes-lui que la princesse sera sienne quand il remplira d'histoires un sac fait de cent coudées de tissu » (C.P. : 82) ; comme toujours dans les contes, la tâche est impossible. Puisque les histoires sont choses immatérielles, comme l'a montré Vladimir Propp (1970: 37-38), l'injonction cache ici une interdiction d'épouser la princesse. Or l'idiot découvre un moyen de contourner l'obstacle. Il se met à raconter « son histoire depuis le début » : « Il dit devant tout le monde ce qu'ils lui avaient donné en échange, et puis il raconta la visite du roi.» (C.P. : 83) Le roi l'interrompt alors, sans doute juste à temps pour éviter son humiliation publique.

Cet épisode, qui constitue une véritable mise en abyme, appelle plusieurs commentaires. Tout d'abord, contrairement à de nombreux contes où c'est le silence qui permet au héros d'échapper au danger, ici c'est l'indiscrétion qui le sauve. Le narrateur ne dit pas si l'indiscrétion fait rire la Cour ou lui fait peur. Tout dépend comment l'on répond à la question suivante : pourquoi le roi n'a-t-il arrêté le récit de l'idiot qu'in extremis? Soit ce roi est un fin politique, et il a voulu savoir quelle faveur ont accordé les trois femmes en échange du lièvre, soit il est si bête qu'il n'a reconnu sa propre histoire qu'au dernier moment. Il va sans dire qu'en ce cas, sa carrière politique est finie. Dans cette hypothèse, l'idiot opère un renversement spectaculaire, car il démontre à la fois sa propre intelligence et l'idiotie du roi. Le roi s'est en effet laissé prendre au fait que l'idiot a annoncé qu'il racontait sa propre histoire et non pas celle du roi et de sa cour. Troisième hypothèse, le roi l'a laissé raconter comme un lecteur curieux, captivé par le récit. Dans cette histoire, le roi serait notre double. Mais prenons garde, lorsque l'on change ainsi de niveau d'analyse, car le narrateur ne raconte pas la même chose que l'idiot, et ainsi le lecteur fictif qu'est le roi ne reçoit pas le même récit que nous. L'idiot raconte à la Cour ce que les trois femmes lui ont donné, mais pas ce qu'a accepté le roi. À l'inverse, le narrateur nous raconte par deux fois l'humiliation du roi (82 et 83), mais il nous frustre, nous lecteurs réels, du secret des femmes. C'est que l'idiot n'a besoin que d'une stratégie politique, tandis que le narrateur vise une composition esthétique du plus haut raffinement. Il construit son récit sur un chiasme, un croisement entre ce que est dit et caché aux uns (les auditeurs fictifs) et aux autres (les lecteurs réels). En outre, le narrateur joue sur les deux tableaux. En apparence, il respecte la bienséance et s'autocensure sur le chapitre des femmes. Mais en insistant sur le mystère de ce "présent de valeur » et en soulignant la différence de traitement que réserve l'idiot aux hommes (le cuisinier et le roi) et aux femmes, le narrateur suggère quelque chose de sexuel. Ici son art de l'ellipse et de l'implicite provoque à coup sûr le rire plus que la frustration. Il réussit le tour de force d'attirer si bien l'attention sur ce qu'il n'a pas dit que tout le monde comprend de quoi il retourne. On voit qu'ici l'art de raconter est inséparable de l'art de se taire. Mais ce conte est encore plus réflexif qu'il n'y paraît, car avec ce mauvais lecteur qu'est le roi, le narrateur nous conseille obliquement d'être prêts à nous demander si l'histoire que l'on nous raconte nous concerne. Or ce conte ne nous parle-t-il pas de nous, lecteurs, du mobile de notre passion pour les contes, qui serait de percer à jour l'intimité des autres et leurs secrets? 


\section{II.2. Le héros sauvé par l'art de se taire}

15 Le secret, l'ellipse, l'implicite, ou au contraire l'indiscrétion, nous mènent tout droit à la question du silence, aussi consubstantiel aux arts de la parole qu'il l'est pour la musique. Le thème du bon usage de la parole et de ses dangers est universel. Mais il n'est guère surprenant de le trouver particulièrement illustré dans le recueil tsigane. La communauté tsigane était, et demeure sans doute, une société fondée sur des stratégies de fermeture et des secrets destinés, entre autres, à se protéger d'un environnement hostile. Jerzy Ficowski, spécialiste polonais des Tsiganes, cite dans un de ses livres d'ethnologie le proverbe tsigane suivant : « Coupe-toi la langue avant que ta langue ne te fasse couper la tête. » (Ficowski, 1989 : 110)

Dans "Les trois cheveux dorés du Roi du soleil», au contraire du conte précédent, le héros est sauvé par l'inversion et l'ellipse. Il s'agit d'un récit très long qui enchaîne sans doute plusieurs contes. Seule la dernière partie concerne notre propos. Un jeune Tsigane pauvre et serviable a réussi grâce à une bonne Ourma (c'est l'équivalent tsigane de Baba Yaga) à épouser la princesse. Mais le roi est opposé à cette mésalliance et lui impose une ultime épreuve : rapporter les trois cheveux dorés du Roi du Soleil. En chemin, le Tsigane promet à un vieux passeur de lui rapporter la solution qui mettra fin au charme qui l'enchaîne à son canot, à une première ville la solution qui lui rendra sa source d'eau de jouvence, et à une seconde ville la solution qui permettra à son pommier de donner de nouveau des pommes qui procurent l'immortalité. La mère du Soleil extorque à son fils, durant son sommeil, les trois cheveux et les réponses aux trois questions, dans l'ordre inverse de leur découverte par le héros. Le héros fait demi-tour et délivre les trois réponses dans l'ordre où elles lui ont été données par la mère du Soleil parce que cet ordre correspond à ses étapes sur le chemin du retour (c'est-à-dire pommier, source, passeur). Cependant le Tsigane a compris un secret que ni le Soleil ni sa mère ne lui ont révélé ou dont le narrateur nous a privés par une ellipse. S'il veut traverser le lac sans rester enchaîné au canot, le héros ne doit révéler la solution au passeur qu'après la traversée. Ce qu'il fait. La première leçon que nous donne ce conte consiste donc à ne parler, ne raconter qu'au moment approprié et à savoir se taire jusque là. Un conseil en or pour les conteurs : savoir à quel moment délivrer une information décisive ou dans quel ordre en délivrer plusieurs. De retour au palais, le Tsigane commence le récit de ses aventures, mais non pas dans l'ordre chronologique. Il commence par le pommier et la source, provoquant chez le roi le désir impérieux de partir sur le champ se procurer l'eau de jouvence et les pommes qui confèrent l'immortalité. Privé de la solution concernant le passeur, le père de la princesse se fait piéger, car il suffit au rameur, pour se désensorceler, de mettre les rames dans les mains de son passager et de sauter hors du canot. Au passage on note que ce conte suggère que certains secrets ne sont que des évidences mises en scène comme des secrets et que le passeur n'était enchainé que par son manque d'initiative ou de bon sens, voire par sa crédulité (il a cru être victime d'un charme). Seconde leçon, le roi est l'exemple même du mauvais lecteur qui est trop impatient pour attendre la fin du récit, la bêtise prend chez lui la forme de la hâte de conclure, et il ne tient pas compte de la mise en garde du héros. Troisième et dernière leçon : il est des cas où le grand art n'est plus de retarder l'information de son auditeur, mais de pratiquer carrément la rétention d'information : ellipse, réticence... 


\section{II.3. Le salut du héros par l'art de lire}

17 Savoir écouter jusqu'au bout aurait sauvé, sinon la vie, du moins le bonheur du roi. Plusieurs contes montrent que savoir lire au sens d'interpréter peut sauver la vie du héros. Bien que la société tsigane ait été, dans le passé, une société sans écriture, cela ne l'empêche pas de valoriser les héros bons déchiffreurs d'énigmes, tels ÆEdipe. Le conte «Les devinettes du petit nain » (R.A.S., conte 23) met en scène l'histoire de quatre frères tsiganes très pauvres. Les trois aînés quittent le foyer les uns après les autres pour chercher fortune. Ils rencontrent sur leur chemin un petit nain qui leur promet nourriture et argent s'ils trouvent le mot d'une énigme. Ils échouent et deviennent les valets du nain. Leur benjamin se lance sur leurs traces, mais, chemin faisant, celui-ci se lie d'amitié avec un ours, un renard et un loup. Lorsqu'il rencontre le petit nain, il trouve la réponse, et comme le nain se met à le frapper, il siffle ses amis animaux qui viennent à la rescousse. Or quelle était la réponse aux quatre énigmes ? À la première, le violon, à la seconde la chaîne (sans doute celle du montreur d'ours), à la troisième les roues de la roulotte. La quatrième se présente comme les précédentes sous la forme d'une courte poésie : « Un serpent long et gris / rampe par terre à l'infini. » À quoi le Tsigane répond : «C'est une route! » La réponse avait donc dans tous les cas un rapport avec l'identité du personnage interrogé, en l'occurrence avec le style de vie nomade des Tsiganes. Les énigmes étaient réflexives, au sens où elles exigeaient de l'interlocuteur de se connaître lui-même, exactement comme l'était l'énigme du Sphynx adressée à đEdipe ${ }^{15}$. À ce stade nous tenons la leçon délivrée aux personnages : elle concerne l'identité tsigane. Mais si nous nous situons au plan des lecteurs réels, ce conte est encore plus intéressant. Le lecteur n'est pas forcément tsigane et cependant il pourrait trouver lui aussi la réponse, du moins la quatrième. En effet, contrairement au héros qui doit puiser dans ses ressources intérieures, l'auditeur/lecteur entend ou lit les trois premières énigmes et leurs réponses, de sorte que parvenu à la dernière, il est en mesure de la résoudre, s'il a correctement dégagé la cohérence thématique des trois précédentes. Dès lors, ce qu'enseigne ce conte n'est pas seulement la conscience de la réflexivité qui permet de déchiffrer à l'unité une énigme ou un seul conte, mais la capacité à lire en série des devinettes ou aussi bien des contes rassemblés en recueil. En outre, ce conte donne les moyens à un gadjo bon lecteur de saisir des secrets de l'identité tsigane, ce qui correspond à l'ouverture assumée à la fin du conte inaugural du Rameau de l'Arbre du soleil, où le narrateur confie qu'il s'est décidé à mettre par écrit les contes qu'il tenait de ses informateurs tsiganes pour ses deux filles et «tous les enfants qui aiment les contes tsiganes » (R.A.S. : 20).

Un autre récit, tiré des Contes de Pologne et intitulé «Devinette » confirme que la forme suprême de l'intelligence est la capacité à percevoir la réflexivité. Un prince se met en tête d'épouser une princesse qui pose des devinettes et fait mourir tous les prétendants qui échouent. En jeune homme prévoyant, le prince réclame à sa propre mère du poison pour se suicider en cas d'échec. Au cours de sa quête, le poison se renverse, tue le cheval, puis les trois corbeaux venus dépecer la charogne, et enfin les douze brigands qui mangent à leur tour les trois corbeaux et meurent empoisonnés. Parvenu au palais, la princesse soumet au héros l'énigme suivante : «[...] trois de un, un de ces trois, trois fois et encore trois. Celui qui résoudra mes questions / m'épousera pour de bon. » (C.P. : 51) À quoi le prince répond: "Trois périrent à cause d'un, de ces trois périrent douze [...] ", puis il résume son histoire pour dégager explicitement à la fois la correspondance entre 
ses chiffres et ceux de la princesse et la logique du conte. Ici, ce n'est plus l'identité du héros qu'il faut reconnaître, mais la structure même du conte. En d'autres termes, si le personnage doit reconnaitre ses propres aventures, le lecteur doit, lui, reconnaître la récapitulation chiffrée et codée du conte qu'il est justement en train de lire. Dans ce cas, la réflexivité du conte ne porte plus seulement sur lui-même pris à l'unité mais sur le genre, car la multiplication par trois plus un des victimes du poison fait directement écho à la structure d'innombrables contes fondée sur des d'épreuves triples ou quadruples.

Il ressort donc de ces analyses, qu'en se greffant parfaitement sur le premier degré, la réflexivité permet de dédoubler la signification sans dédoubler l'action. C'est ce procédé de construction qui permet à l'art du second degré d'être accessible, au moins de manière intuitive et inconsciente, au public enfantin ou populaire et de n'être pas l'apanage d'une lecture savante. Il est d'ailleurs révélateur que la dimension réflexive du conte « $\mathrm{La}$ Chèvre de Chelm " soit présente dans l'édition pour enfants de l'École des loisirs mais absente dans la version tout public du recueil de Ben Zimet, sous le titre «La Chèvre et le bouc» (Zimet, 2000 : 90-97). Nos contes privilégient deux formes d'intelligence: tout d'abord, la retenue qui consiste à raconter ou à se taire à bon escient, à laisser une place à l'auditeur grâce aux manques, aux lacunes, d'un genre dont la force réside, selon Walter Benjamin, dans "le refus d'explication"; en second lieu, la capacité à détecter la réflexivité et le double sens, à comprendre que l'on est concerné, que la réalité est concernée, par une histoire, fût-elle en apparence invraisemblable, parce que le conte emploie un langage symbolique.

\section{Réflexivité et dimension initiatique : de l'art à la vie et retour?}

\section{III.1. Interprétation du langage symbolique et maturité sexuelle}

La réflexivité de nos contes ne relève peut-être pas uniquement du fonctionnement autoréférentiel de la littérature. Son enseignement concerne également la vie. En effet, ce n'est peut-être pas un hasard si, dans le conte yiddish " La Chèvre de Chelm », les mauvais lecteurs (les deux hassidim ainsi que le couple formé par le rabbin et sa femme Malka) sont incapables de distinguer une chèvre d'un bouc, c'est-à-dire de reconnaître la différence des sexes. La capacité de conter, se taire ou interpréter, a assurément des rapports avec la maturité sexuelle et la connaissance des secrets qui régissent les relations de séduction ou la vie de couple. On se rappelle le chapitre de Bruno Bettelheim, consacré au conte des frères Grimm, «Les Trois plumes » $\left(2009, \mathrm{n}^{\circ} 63 ; 1812\right.$, KHM 64) et intitulé «Simplet, le benjamin de la famille» (1976, I, chap.16: 165-173). Le psychanalyste explique l'un des paradoxes dont il faut tenir compte pour articuler réflexivité et initiation à la sexualité et nuancer l'équation du conte yiddish entre maturité, intelligence et mécréance. Le héros qui triomphe n'est pas nécessairement le plus intelligent, au départ ou en apparence. Dans les contes, c'est souvent le benjamin, présenté comme idiot, qui réussit. Bruno Bettelheim avance l'hypothèse que celui-ci réussit parce qu'il s'appuie sur son inconscient. À l'examen des contes de notre corpus, on peut préciser que la fortune sourit tantôt à ceux qui savent lire dans leur propre inconscient tantôt à ceux qui savent lire dans l'inconscient d'autrui (beaux-parents, femme, auditeur/lecteur) pour le satisfaire ou le manipuler. Dans tous les cas, le succès est lié à une compréhension du langage symbolique et de l'inconscient, et pas uniquement 
aux formes rationnelles du savoir. "Le vaillant petit tailleur ", vingtième conte des frères Grimm, propose, pour ainsi dire, un répertoire complet des bons et des mauvais usages de cette connaissance qui enchanterait les psychanalystes. Ce frêle héros s'impose grâce à un malentendu. Ayant tué sept mouches d'un seul coup de torchon, il brode sur sa ceinture : "Sept d'un coup». Laissant planer ainsi le doute, il suscite la crainte, car tous ses interlocuteurs croient qu'il a tué sept hommes d'un coup. Après une première série d'épreuves, à l'issue desquelles il a échappé à une tentative de meurtre de la part du géant, il parvient dans la cour d'un palais où il s'endort. L'invraisemblance ne se limite pas au comportement extrêmement sans-gêne du héros. L'épisode suggère qu'il est des moments où au lieu de déployer des efforts, il vaut mieux laisser travailler autrui ou notre inconscient pour nous. Durant son sommeil, des courtisans lisent l'inscription de sa ceinture et conseillent au roi de l'enrôler dans son armée plutôt que de s'en faire un ennemi. Il s'en suit une seconde série d'épreuves, par lesquelles le roi, empreint de mauvaise foi, tente de se débarrasser de ce dangereux rival. Le vaillant petit tailleur triomphe malgré tout, notamment de deux épreuves symboliquement contradictoires : il vient successivement à bout d'une licorne, traditionnellement symbole de pureté et de chasteté, et d'un sanglier, symbole des pulsions qu'il faut contrôler.

Or ses aventures ne se terminent pas avec l'ascension sociale et le mariage du héros. Deux ultimes épisodes font intervenir le sommeil. Peu après son mariage, le tailleur parle dans son sommeil et trahit sa basse extraction sociale. Son épouse et son beau-père ourdissent une tentative de meurtre qu'il déjoue la nuit suivante, en feignant, cette fois-ci, de parler dans son sommeil et en tenant des propos menaçants contre ses agresseurs, leur signifiant qu'il les a découverts. Si l'on tente d'interpréter ce conte autrement qu'une farce ${ }^{16}$, on pourrait résumer la leçon comme un superbe dispositif dialectique mettant en garde ses lecteurs contre des dangers contradictoires : le premier épisode, où le vaillant petit tailleur dort, nous incite à faire confiance à notre inconscient, parce qu'il puise notamment aux formidables sources d'énergie de la libido, à penser que l'on peut parfois réussir sans s'épuiser au travail (conscient), ce qui contrevient à la morale courante et à l'éthique protestante du travail, l'avant-dernier épisode rappelle que malgré tout l'on peut être trahi par son inconscient (les mots prononcés dans son sommeil pourraient en effet s'apparenter à un lapsus), et le dernier suggère que le comble de la ruse consiste parfois à mimer la logique de l'inconscient, c'est-à-dire la spontanéité, la sincérité, pour mieux tromper ses adversaires. Dans ce conte, le héros serait le champion du déchiffrement de son inconscient et surtout de celui d'autrui dont il perce à jour la crédulité, la puissance de l'imaginaire qui leur fait croire à sa capacité de nuire sans un commencement de preuve. En d'autres termes, il a tout compris de la distinction philosophique entre puissance (effective) et pouvoir (imaginaire) et en a tiré toutes les conséquences politiques. La variante polonaise du vaillant petit tailleur confirme que le héros "peu prometteur", pour reprendre la terminologie de Nicole Belmont, triomphe grâce à sa capacité d'interprétation du langage symbolique qui va de pair avec sa compréhension des secrets sexuels. Dans "Wojtek le puissant», le héros est lui aussi soumis à une ultime épreuve, après avoir triomphé de toutes les précédentes. Le roi, qui préfèrerait accorder la main de sa fille au géant Kuba, leur prescrit l'épreuve suivante : "Cette nuit, vous allez dormir sur une seule couche: Kuba, Wojtek et la princesse. La princesse dormira au milieu de vous deux. Celui vers lequel elle tournera son visage l'épousera. » (C.P. : 120) Or Wojtek se tire d'embarras en plaçant entre Kuba et la princesse un baluchon malodorant, ce qui détourne la jeune fille de son rival et oblige le roi à faire 
du tailleur son gendre. Tout se passe comme si Wojtek avait réussi à déchiffrer correctement le sens sexuel de l'injonction du roi dont la préoccupation majeure est peutêtre de s'assurer une descendance plus encore que de contracter une alliance flatteuse et rassurante avec le géant Kuba. En effet, « l'odeur très désagréable » que semble dégager Kuba amène la princesse à se tourner face à Wojtek, c'est-à-dire du bon côté pour avoir une sexualité féconde. Tourner le dos eût été s'offrir à la sodomie, sexualité dissociée de la procréation, l'odeur nauséabonde étant l'indice d'un jeu entre sexualité génitale et anale. On serait cependant tenté de nuancer en soulignant que si Wojtek parvient à ses fins, l'excipit n'évoque ici ni la joie des noces ni le bonheur conjugal. La tentation est alors de se rabattre sur une lecture morale selon laquelle ce conte adresserait une mise en garde aux ambitieux en suggérant que réussite sociale et bonheur amoureux ne coïncident pas forcément. Peut-être aussi l'initiation de Wojtek est-elle inachevée, car s'il a su déchiffrer le désir de son beau-père, il n'a pas pris en compte celui de sa future femme.

\section{III.2. Déchiffrer le désir des femmes}

Différents contes suggèrent que pour séduire une femme, il faut être capable de déchiffrer et satisfaire son désir inconscient. Trois contes présentant des motifs communs donnent un aperçu de la manière dont le héros répond à la question freudienne par excellence : que veut la femme? Il s'agit du " Miroir qui voyait tout » (R.A.S., conte 17), de « Hélène la Magique I» (C.P.R., t. 2, conte 181) et « Hélène la Magique II » (C.P.R., t. 2, conte 182). Il est difficile de déterminer s'il existe entre le conte tsigane et les deux variantes russes une relation d'influence ou un simple parallèle attestant de l'universalité du conte. Du point de vue de la structure, le conte tsigane se présente comme un conte simple, tandis que les deux contes d'Afanassiev présentent une structure complexe, comme s'il s'agissait de l'enchaînement de deux contes dont seul le second présente une ressemblance avec le conte collecté par Jerzy Ficowski.

Dans les trois contes, le héros (un valet de ferme tsigane ou un simple soldat russe) est soumis au même genre d'épreuve pour épouser la femme qu'il désire. Il doit trouver une cachette qui le rend introuvable. Dans "Le Miroir qui voyait tout ", l'injonction émane des parents de la princesse. Le héros est ainsi confronté à une épreuve insurmontable, premièrement parce que la princesse possède un miroir magique qui voit tout, et deuxièmement, parce que l'injonction est contradictoire : si le Tsigane se cache trop bien, jamais la princesse ne le retrouvera et ainsi ils ne se marieront jamais. En d'autres termes, les parents de la princesse lui ordonnent de disparaitre à tout jamais et refusent en fait de laisser leur fille se marier. Le conte ne précise pas si c'est par crainte de la mésalliance ou en raison d'attachements familiaux excessifs. On reconnait une fois de plus la pertinence de l'analyse de Wladimir Propp qui identifie l'une des étapes incontournables du conte par la formule «Le héros se fait signifier une interdiction» (1970: 37), en précisant quelques lignes plus loin que l'interdiction peut prendre la forme d'une injonction. Or le Tsigane, à chaque fois qu'il se cache, grâce à un objet magique, se trahit parce que le pompon de son bonnet dépasse. Le pompon qui dépasse exerce assurément une séduction du fait qu'il n'est pas exempt de connotation sexuelle mais que celle-ci est euphémisée par le caractère enfantin et comique de cet appendice. D'ailleurs, le héros se trahit involontairement, ce que l'on peut interpréter comme le gage de la sincérité de son désir 
et comme le fait que c'est son inconscient et non pas sa raison qui vient à bout de la contradiction contenue dans l'injonction. C'est ici qu'intervient le miroir.

Ce miroir «qui voit tout » empêche littéralement la princesse de se marier et remplit la même fonction que des parents hostiles ou ceux-ci sous la forme intériorisée du Surmoi de l'héroïne. Cependant, le miroir est aussi une image de la réflexivité et ici, en l'occurrence, il constitue peut-être une indication de lecture pour le conte lui-même. En effet, que montre un miroir? Le reflet inversé de la réalité. Aussi le héros semble-t-il avoir compris qu'il faut inverser l'injonction parentale, la lire comme un reflet dans un miroir, pour parvenir à ses fins : «Si tu veux l'épouser, tu ne dois pas trop bien te cacher. Il faut qu'elle puisse te retrouver. » À la fin du conte, le miroir retrouve momentanément l'une de ses significations traditionnelles, «il reflète le contenu du cœur et de la conscience » (Chevalier \& Gheerbrant, 1982:636) et dévoile ainsi à la princesse que «le Tzigane s'est installé dans [s] on cœur ${ }^{17}$ " (R.A.S. : 118). Mais malgré ce rôle positif, le miroir refuse de lui indiquer où est caché physiquement le jeune homme, si bien que la jeune fille brise son miroir magique, ce qui suffit à faire sortir le héros de sa cachette. Si le miroir est comme l'œil des parents omniscients, lisant dans nos pensées et contrôlant notre vie, ainsi que l'imaginent fréquemment les enfants, le fait que la princesse brise celui-ci suggère qu'elle est parvenue à rompre ses attachements œdipiens et à mettre suffisamment de distance entre elle et ses parents pour protéger l'intimité de son couple. Le héros tsigane, qui s'est montré assez clairvoyant pour braver l'interdiction de ses futurs beaux-parents, avait déjà accompli son émancipation en quittant ses premières attaches, son logis et son village. Il a aidé la princesse à se libérer de ce qui aliénait son désir (l'obéissance à ses parents et la transparence à leur égard). Il l'a séduite aussi par son humour et la promesse d'une liberté au sein de leur couple en lui disant, entre sa première et sa seconde épreuve : «[...] si tu me trouves, tu feras de moi ce qui te plaira. » ( R.A.S. : 117) Cette parole est en effet à double sens, car elle peut signifier, sur le plan conscient, qu'il acceptera son châtiment, et sur le plan inconscient, qu'il lui laissera l'initiative dans le domaine érotique. Ce conte indique donc qu'il faut prendre en compte le désir de sa partenaire, mais que les contradictions entre son désir conscient et son désir inconscient peuvent rendre son déchiffrement compliqué.

Dans les deux contes russes, la question de la lecture au sens d'interprétation devient plus explicite du fait que certains personnages sont dotés d'un livre. Dans «Hélène la Magique II ", le miroir joue à peu près le même rôle que dans « Le Miroir qui voyait tout ", tandis que c'est un livre de sorcellerie qui remplit cette fonction dans "Hélène la Magique I ». C'est entre « Le Miroir qui voyait tout » et « Hélène la Magique II » ${ }^{18}$ que l'on observe la distance maximale. Hélène la Magique n'est pas une timide et pure jeune fille. C'est une femme faite, apparemment libre de tous liens familiaux, aussi savante que belle. Comme l'explique la traductrice Lise Gruel-Apert en note (C.P.R., t. $2: 402$ ), sa « voix tonnante » est l'indice qu'elle est douée de pouvoirs magiques. Point décisif, elle paraitt insensible à la pitié et à l'amour : elle fait décapiter les onze prétendants qui ont échoué aux épreuves qu'elle leur a imposées et ce n'est pas par sollicitude pour le héros qu'elle brise son miroir magique mais par colère d'être vaincue et obligée de l'épouser. Contrairement au dénouement euphorique du conte tsigane, ici point de conversion à l'amour ni de joie.

En première lecture, on a l'impression d'un chiasme par rapport au conte tsigane : c'est le héros qui semble entravé par des affects œdipiens. Tout d'abord, en s'éprenant d'Hélène la Magique, il désire la femme que son père adoptif, le Dragon, a poursuivie de ses 
assiduités durant trente-trois ans (ce qui suggère qu'Hélène la Magique est bien trop vieille pour notre héros ou qu'en bonne sorcière, elle ne vieillit pas). En second lieu, à peine a-t-il capturé Hélène la Magique par la ruse et l'a-t-il installée chez ses parents, qu'il confie à sa propre mère la clé du coffre où sont enfermées les ailes de celle-là. Par un acte manqué parfaitement réussi du point de vue de l'inconscient, la mère du héros rend ses ailes à la compagne de son fils et lui permet ainsi de s'enfuir.

Commence alors la seconde partie du conte, où le héros se lance à la reconquête d'Hélène la Magique. Cette fois-ci, il doit «se cacher trois fois sans être découvert par elle » s'il veut échapper à la mort et l'épouser. Curieusement, la belle et savante Hélène ne se radoucit pas, une fois qu'il a triomphé de ses épreuves et qu'elle a brisé son miroir. Ce dénouement suggère qu'il ne suffit pas de s'affranchir de ses parents pour séduire une femme. Par ailleurs, le lecteur se demande pourquoi à chaque étape le héros a préféré Hélène la Magique à sa servante qui, elle, au contraire de sa maîtresse, aide le héros et s'inscrit dans une relation de réciprocité (il la libère du dragon ; elle lui révèle la cachette qui lui permet de triompher). Le choix entre les deux femmes, de conditions sociales très différentes, est symbolisé par des paires d'objets, l'un en or, l'autre en argent. Le héros choisit toujours l'or associé à Hélène la Magique et néglige l'argent associé à la servante. Choix d'un ambitieux qui sera puni par le manque d'amour de son épouse? Ou choix surmoïque de la relation amoureuse la plus flatteuse? Notre héros serait-il un mauvais déchiffreur de son désir et du désir des femmes?

Si l'on prend en compte l'idée, plutôt jungienne, selon laquelle on ne rêve jamais que de soi et que le conte, comme le rêve, nous présente non pas des personnages véritablement distincts mais un seul et même personnage diffracté en ses différentes instances psychiques, on pourrait faire l'hypothèse que le quatuor (héros et Dragon d'une part, Hélène la Magique et servante d'autre part) se réduit à un duo de personnages clivés par un conflit interne. Ainsi Hélène la Magique serait-elle le Surmoi, tyranniquement exigeant et jamais satisfait, de la partenaire féminine, tandis que la servante serait la part du Moi qui, en elle, a envie d'avoir une vie amoureuse et de se marier. Dans ce cas, l'issue peu satisfaisante du conte indique que la solution ne dépend pas entièrement de la maturation psychique du personnage masculin, mais aussi de la résolution des conflits internes du personnage féminin.

Le héros a-t-il accompli quant à lui tout le parcours qu'il devait et quel rôle joue le « livre énorme » du Dragon dans la première moitié du conte ? Profitant du sommeil du Dragon, le héros lit dans ce livre la maxime suivante: « Un tsar ne peut mettre au monde un tsar, seule le peut une tsarine.» (C.P.R., t. $2: 348$ ) Saisi d'une intuition inexpliquée, il corrige : "Une tsarine ne peut mettre au monde un tsar, seul le peut un tsar.» (C.P.R., t. $2:$ 348) C'est la lecture de cette phrase, complétée par une explication orale d'Ivan, qui convainc le Dragon qu'il vient d'engendrer à lui seul un fils qui n'est autre que notre héros: "Quand tu t'es retourné et que tu as pété, j'ai sauté hors de toi [...].» (C.P.R., t. $2: 349)$ En d'autres termes, le héros manipule le Dragon prêt à admettre ce que d'aucuns appelleraient des «théories infantiles » concernant la procréation : successivement la parthénogénèse, puis l'androgénèse couplée à la théorie de l'enfant-pet, version euphémisée de l'enfant-excrément. La naïveté infantile du Dragon accrédite davantage l'idée qu'il représente une instance psychique du héros (son ça, ses pulsions et fantasmes archaïques...) que celle qu'il serait son père adoptif. La supériorité intellectuelle d'Ivan ferait de ce dernier une figure du Surmoi. La preuve en est que le Dragon n'est pas jaloux, mais qu'il l'aide et le met en garde : « [...] je te plains car Hélène la Magique en sait long. » 
Cette relation entre le Dragon et Ivan indique que le Surmoi ne peut se passer de l'énergie libidinale du ça pour conquérir une femme, fût-elle savante. Mais, bien qu'il ait dépassé ses théories infantiles et accepté de coopérer avec sa part pulsionnelle et archaïque incarnée par le Dragon, la préférence d'Ivan pour l'or et Hélène la Magique trahit peutêtre une intégration inachevée des différentes instances psychiques.

Sa maturation a commencé avec un mensonge (une imposture, une filiation usurpée), mais aussi avec un art consommé de la réécriture. Il lui suffit de changer quelques lettres pour inverser le sens de la phrase : «Il gratta ces mots avec la pointe d'un canif et écrivit à la place [...]. » (C.P.R., t. $2: 349)$ Il trahit ainsi une véritable indépendance d'esprit face à l'écrit, contrairement au Dragon qui se laisse abuser par l'inscription, tout comme les adversaires du vaillant petit tailleur - victime d'un trop grand respect de l'écrit ? - et prend un chemin qui mène de la lecture à l'écriture, de la passivité à l'activité. Mais il s'agit de réécriture, ce qui fait de cet épisode une véritable indication réflexive. La présence de deux variantes d'Hélène la Magique dans le seul recueil d'Afanassiev - sans parler de la version tsigane - n'est-elle pas la preuve que la lettre du conte, voire son esprit, ne sont pas sacrés, que le texte peut être retouché, voire inversé, et que c'est en vertu de ce procédé et de cette liberté de création qu'il convient de lire ce "Hélène la Magique II »? En d'autres termes, le héros Ivan n'est pas seulement un fin connaisseur de son inconscient ou de celui d'autrui, mais une figure du conteur en créateur de variantes, qui révèle, pour qui sait l'entendre, les secrets de son art.

\section{III.3. Lucidité du conteur sur son lecteur et le genre du conte}

31 Comme nous l'avons vu plus haut, Ivan, le héros de "Si ça ne te plaît pas, n'écoute pas » a gagné quatre-vingt dix roubles en faisant les poches des clients du marché. Sur le chemin du retour, il défie «le seigneur du lieu » en lui promettant de gagner à ses dépens deux cents roubles : "Oui, mais parions d'abord: si j'arrive à te faire dire "Tu mens !", tu me dois deux cents roubles; si je n'y arrive pas, tu es libre de faire de moi ce que tu voudras." (C.P.R., t. $2:$ 296) Il s'en suit une improvisation drôle et brillante d'Ivan, introduite par le narrateur en ces termes: «[...] et Ivan se lança dans un conte [...]» (296). Ce conte enchâssé qui occupe l'essentiel du texte finit par avoir raison de la résistance du seigneur qui l'écoute et lâche les deux mots fatals. On peut invoquer la longueur de ce conte, l'effet d'accumulation et les incohérences pour expliquer qu'Ivan fasse oublier le début et les clauses du pari à son auditeur. Cependant, le dénouement révèle de manière elliptique un art consommé de la manipulation qui repose sur une prévision parfaite de la réception du récit.

La corde finit par casser et j'atterris droit dans les enfers. Ah, si j'ai eu du mal à en ressortir! Eh bien, Maître, sais-tu qui j'ai vu là-bas? Ton père qui servait de bourricot à charrier le fumier... - Tu mens, imbécile ! (297)

Ivan exploite les points faibles du seigneur: sa piété filiale et son sens de l'honneur aristocratique qui lui interdisent de laisser bafouer la mémoire de son père, sa foi qui lui fait craindre pour le salut de son père, sa morgue sociale qui lui rend insupportable la vision d'un père commis à une tâche humiliante et rabaissé au rang d'un animal, en une illustration spectaculaire de la promesse biblique «les premiers seront les derniers », et peut-être la nécessité de refouler ses doutes concernant la moralité du défunt, que trahirait la violence de sa réplique. Le texte, par sa composition faussement circulaire, nous rappelle furtivement, dans l'incipit, qu'Ivan vient de perdre son propre père et que le seigneur qui est dans la même situation est, dans l'excipit, pour ainsi dire son double. 
Cependant, la tonalité de la fin du récit d'Ivan suggère que celui-ci n'a pas puisé dans son expérience la moindre capacité d'identification et d'empathie avec un autre fils endeuillé, mais une audace sacrilège (œdipienne ?) à l'égard des pères morts. Tout indique en effet que la mort de son propre père l'autorise à transgresser et jouir sans entrave.

Pour autant, faut-il n'attribuer le triomphe d'Ivan qu'à la manipulation psychologique de son auditeur? Son succès ne doit-il rien à la séduction esthétique du conte qu'il improvise et qui se présente sous la forme d'une fatrasie jubilatoire? Il serait en effet assez superficiel de taxer Ivan d'incompétence du fait que son récit accumule les adunata et les contradictions. Son talent n'est en rien inférieur à celui du narrateur en titre. Son délire verbal, comparable au génial récit que fait Pinocchio à son père Gepetto, lorsqu'ils se retrouvent dans le ventre de la baleine, mériterait une analyse détaillée de ses procédés comiques, de sa poésie onirique. Il propose, dans la foulée, une brillante réflexion sur le genre même du conte. Le « Tu mens » final ne concerne pas uniquement le défunt père du seigneur. Il résonne aussi comme une protestation contre les excès du conte : jusqu'où ce genre, fort peu réaliste, peut-il aller dans le domaine de l'invraisemblance, sans ruiner la suspension bénévole du jugement critique et du rationalisme du lecteur? Les invraisemblances font certes partie intégrante du conte, mais elles ne doivent jamais nuire à sa cohérence symbolique. Le récit d'Ivan, au contraire, est une parodie de cette esthétique du merveilleux dont la seule cohérence serait sa signification réflexive : outre l'accumulation parodique, Ivan inverse un motif traditionnel du conte. Il remet les entrailles dans le cadavre du cheval au lieu de le vider et de se fourrer dans sa peau. Ou encore il dévoile le procédé de composition du conte enchâssant lorsqu'il raconte comment il redescend du ciel :

Je ramassai de la poussière et de la suie, j'en tissai une corde, je l'attachai à un nuage et je commençai à descendre. Je descendis, je descendis jusqu'au bout de la corde. Arrivé là, j'eus une idée : couper le haut de la corde, le rajouter au bas. Je coupe le haut, je le rajoute au bas, je recoupe, je rajoute, je recoupe, je rajoute, et je descends. (297)

Ce principe de déconstruction qui consiste à couper le haut et à le rajouter au bas est très exactement l'inverse de ce qu'a fait le conteur en titre, lorsqu'il a déplacé et inversé le motif traditionnel d'excipit (le conteur privé de vodka et de miel) pour en faire, dès le troisième paragraphe du conte celui d'Ivan « trempant son pain d'épice dans le miel ». Le pêle-mêle qu'il nous propose fait assurément écho à d'autres contes du répertoire russe, voire du recueil d'Afanassiev, et montre que le conte populaire est non seulement capable de réfléchir sur sa propre poétique mais éventuellement de penser contre lui-même, de proposer une parodie de son illogisme et de son recours au surnaturel. En ce sens, le narrateur du conte délègue narrateur secondaire, en l'occurrence Ivan, une tâche plus brillante que la sienne et se cantonne, quant à lui, à sa posture traditionnelle de serviteur médiocre - entendons ici modeste - d'un genre mineur.

Le second degré dont la réflexivité n'est qu'une forme n'est donc ni l'apanage de la littérature savante ni une invention de la littérature pour la jeunesse contemporaine dont il constitue, il est vrai, l'une des tendances majeures. Il est déjà présent dans les contes populaires pour adultes collectés au XIX ${ }^{\mathrm{e}}$ siècle en Europe centrale et orientale. L'analyse a mis en lumière que c'est en greffant le second degré sur le premier, en conférant à la dimension réflexive une fonction dramatique centrale dans l'intrigue, que ce raffinement littéraire devient accessible tant au public populaire de jadis qu'aux jeunes lecteurs d'aujourd'hui. Il apparaît aussi que la réflexivité a des rapports avec la maturité sexuelle 
des personnages, des conteurs, et par voie de conséquence des lecteurs. Cela n'empêche aucunement les contes en question d'être appréciés des enfants, car le contenu initiatique est toujours voilé par un langage symbolique et imagé qui ménage et la pudeur et l'immaturité de ce public. Le sens des contes se révèle rarement à la première lecture, dans l'enfance, mais il est réinterprété tout au long de la vie, travaillant souterrainement, dans l'inconscient des lecteurs. Aussi convient-il de laisser à l'excipit d'un conte, le très fameux « Hansel et Gretel » des frères Grimm, le dernier mot :

Mon conte est fini, là-bas court une souris, et qui l'attrapera, un très, très grand

bonnet de fourrure s'en fera. (Grimm, 2009 : 104)

Cette image drôle et modeste dit, entre autres, que, pour qui sait lire, « la peau de souris » d'un genre mineur recèle une sagesse d'une richesse insoupçonnée.

\section{BIBLIOGRAPHIE}

AARne Antti \& THOMPson Stith (1961), The Types of the Folktale, FFC nº 184, Helsinki.

AfANASSIEV Alexandre (2010), Contes populaires russes (Narodnye Russkie Skazki), traduit et présenté par Lise Gruel-Apert, Paris : Imago, 3 vol.

Belmont Nicole (1999), Poétique du conte. Essai sur le conte de tradition orale, Paris : Gallimard.

BENJAMIN Walter (2011), Expérience et pauvreté, suivi de Le Conteur. Considérations sur l'œuvre de Nicolas Leskov (1936), et La Tâche du traducteur, traduit de l'allemand par Cédric Cohen Skalli, Paris : Payot \& Rivages.

Bettelheim Bruno (1976), La Psychanalyse des contes de fées, traduit de l'anglais par Théo Carlier, Paris : Robert Laffont.

CHEVALIER Jean \& GHEERBRANT Alain (1969), Dictionnaire des symboles, éd. revue et corrigée en 1982, Paris : Robert Laffont/Jupiter.

Contes de Pologne (1990), racontés par Oldřich Sirovátka, adaptation française de Claude Clément, illustrations de Dagmar Berková, Paris : Gründ [édition originale, Prague : Artia, 1989].

Contes polonais : Maciek et Wojtek (2007), choisis, traduits et adaptés par Agnieszka Macias, illustrations de Mette Ivers, Paris : L'École des loisirs.

Contes yiddish de Chelm à Varsovie (2000), contes choisis et traduits par Sarah Schulmann, Paris : L'École des loisirs.

FICOWSKI Jerzy (1989), The Gypsies in Poland - History and Customs [Dzieje i obyczaje], Varsovie : Interpress Publishers.

FICOWSKI Jerzy (1990), Le Rameau de l'arbre du soleil. Contes tziganes polonais d'après la tradition orale, traduit du polonais et illustré par Małgorzata Sadowska-Daguin, préface de Robert Escarpit, Chateauneuf : Wallâda.

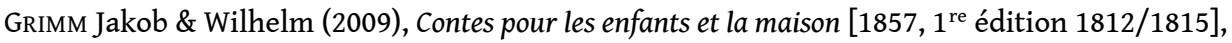
collectés par les frères Grimm, édités et traduits par Natacha Rimasson-Fertin, Paris : éditions José Corti, tomes 1 et 2 . 
GRUEL-APERT Lise (1995), La Tradition orale russe, Paris : PUF.

PenNART Geoffroy de (1998), Le Loup sentimental, Paris : L'École des loisirs.

Potel Jean-Yves (dir.) (2013), « Papusza : poétesse tsigane et polonaise », Études tsiganes, 48-49.

PRopp Vladimir (1970), Morphologie du conte, traduit du russe par Marguerite Derrida, Tzvetan

Todorov et Claude Khan, Paris : Seuil.

Zimet Ben (2000), Contes du yiddishland. Paroles du peuple juif, Paris : Seuil.

\section{NOTES}

1. J'abrègerai désormais en C.P.R.

2. Conte $\mathrm{n}^{\circ} 20$ dans Grimm (2009) et KHM 20 dans l'édition de 1812.

3. La chèvre et ses sept chevreaux renvoient au conte «Le loup et les sept chevreaux » des frères Grimm ( ${ }^{\circ} 5$ dans Grimm, 2009 et KHM 5 dans l'édition de 1812), le Petit Chaperon rouge et le Petit Poucet viennent des contes de Perrault, le conte « Les trois petits cochons » s'enracine dans la tradition anglaise et « Pierre et le Loup » est un conte musical du compositeur russe Sergueï Prokofiev (1936).

4. J'abrègerai désormais en C.D.P.

5. J'abrègerai désormais en C.P.

6. J'abrègerai désormais en C.Y.

7. Le point de vue des littéraires, voire des historiens, qui consiste à intégrer l'Allemagne à l'Europe centrale, pourrait évidemment être contesté par les économistes ou les politologues.

8. J'abrègerai désormais en R.A.S.

9. C'est moi qui souligne.

10. Ce sujet mériterait une étude systématique.

11. Cet excipit, ici présent sous une forme très développée, ailleurs sous forme fragmentaire (voir par exemple C.D.P., contes $\mathrm{n}^{\circ} 5: 40$ et $\mathrm{n}^{\circ} 37: 164$ ou C.P.R., t. 3 , contes $\mathrm{n}^{\text {os }} 194,211,217,219,225$, $240,265)$ est en réalité un sujet de conte à part entière dans la classification internationale d'Aarne et Thompson (AT 1880 « Le conteur rentre du banquet »).

12. Dans ce passage, le narrateur parodie les nombreux contes juifs, notamment ceux de la tradition mystique, où les références à la Torah sont sérieuses et sans doute pertinentes.

13. Je reprends ici la terminologie du schéma initiatique selon Nicole Belmont (1999, chap. 5 : 175), qui s'écarte légèrement de celle d'Algirdas Julien Greimas, en renommant la troisième série « identificatoire » au lieu de « glorifiante ».

14. Le nombre des épreuves obéit ici à une progression géométrique : 1 , puis 3 puis 6 épreuves.

15. Édipe avait compris que la réponse à l'énigme du Sphinx («qui marche à quatre pattes le matin, sur deux à midi et sur trois le soir ?») était "l'Homme », mais il aurait peut-être dû, pour échapper à son sort tragique, pousser plus loin et répondre « C'est moi ».

16. La traduction de ce conte par Natacha Rimasson-Fertin est jubilatoire par son rythme et sa verve comique et justifie qu'elle reprenne à son compte la tradition critique qui le considère comme une farce (Grimm, 2009, t.1, notice: 137). Mais je propose ici de parier sur une signification sérieuse et complexe sous le comique.

17. Les éditions Wallâda ont choisi la graphie de « Tzigane », inhabituelle en français, plutôt que «Tsigane », en hommage aux victimes déportées et marquées au bras du « $\mathrm{z}$ » de Zigeuner. Je respecte leur orthographe dans les citations.

18. Faute de place, on laissera ici de côté l'analyse de « Hélène la Magique I ». 


\section{RÉSUMÉS}

Dans cet article, le second degré est entendu au sens de réflexivité, soit tout ce par quoi la littérature fait référence à elle-même et non pas au monde : l'art de conter, de se taire qui en est inséparable, ou de lire au sens d'interpréter. L'étude de contes d'Europe centrale et orientale (contes de Grimm, d'Afanassiev, contes polonais, yiddish et tsiganes polonais) démontre que la réflexivité n'est pas une invention de la littérature pour la jeunesse contemporaine, bien qu'elle soit l'une de ses caractéristiques majeures, ni l'apanage de la littérature savante. Elle est déjà présente dans les contes populaires pour adultes ou pour «tout public». Cependant, l'intelligence réflexive des personnages, du narrateur ou du lecteur ont un rapport avec la maturité psychologique et sexuelle qui est beaucoup plus marquée dans les contes populaires que dans les textes destinés à la jeunesse. L'analyse porte d'abord sur les procédés d'insertion de la réflexivité, tantôt à la périphérie des contes, tantôt au cœur même de l'intrigue principale. En second lieu, il s'agit de s'interroger sur le sens de cette réflexivité, qui, dans la mesure où elle a partie liée avec une compréhension de l'Inconscient, ne procède pas d'un fonctionnement purement auto-référentiel de la littérature, mais a pour effet, sinon pour but, de nous ramener au monde et de nous proposer une sagesse éloignée de toute bien-pensance.

In this paper, the second degree is construed as reflexivity, that is what makes literature refer to itself as opposed to the outside world: the art of story-telling, of keeping quiet which is part and parcel of it, or the art of reading understood as interpreting. The study of Central and Eastern European tales (tales by Grimm and Afanassiev, as well as Polish, Yiddish and Polish-Gipsy ones) demonstrates that reflexivity is not an invention of contemporary literature for young people, even though this is one of its major characteristics, nor is it an exclusive feature of learned literature. Reflexivity is already present in folk tales for adults or for the "general public". Yet, the reflexive intelligence of the characters, the narrator or the reader, bears a strikingly deeper relation to emotional and sexual maturity in folk tales than in texts intended for the young public. The analysis will first focus on the devices used to insert reflexive elements either into the periphery of the tales or even the core of the main plot. In a second part, the paper will raise the issue of the meaning of such reflexivity which, insofar as it is linked with the deciphering of the unconscious, does not denote a purely self-referential functioning of literature but, on the contrary, brings us back, unwittingly perhaps, to the real world, to formulate wise conclusions that may be far from ethical correction.

\section{INDEX}

Mots-clés : contes populaires, polonais, russe, yiddish, tsigane, Grimm, second degré, réflexivité, littérature pour la jeunesse, conte initiatique, maturité sexuelle, explicite, implicite

Keywords : Polish, Russian, Yiddish and Gipsy folk tales, Grimm, second degree, reflexivity, literature for young people, initiation tale, sexual maturity, explicit, implicit 
AUTEUR

ANNE-MARIE MONLUÇON

Centre LITT\&ARTS (anciennement Traverses 19-21, groupe ECRIRE), Université Grenoble Alpes 